\title{
$342 \quad$ A Wavelet-Transform Based Asker Identification System for Smart Multi-point Tele-conferences
}

\author{
Jinshan TANG, Ryohei NAKATSU ${ }^{\circ}$, Shinjiro KAWATO, Jun OHYA \\ (ATR Media Integration \& Communications Research Laboratories \\ 2-2 Hikaridai, Seika-cho, Soraku-gun, Kyoto 619-0288 Japan) \\ E-mail: \{stang,nakatsu,skawato,ohya\}@mic.atr.co.jp
}

\begin{abstract}
This paper proposes a wavelet-based asker identification system for smart multi-point tele-conferences. Our system is composed of three processors. The first processor is called an "ask" action detector, which is used to decide whether an "ask" action is occurring. If an "ask" action is detected. the input image is sent to the face detector that detects the human face from the complex background. The detected lace is fed to a wavelet-based face identification processor to recognize the asker.
\end{abstract}

Key words multi-point tele-conference, wavelet, asker detection, face recognition

\section{Introduction}

In smart multi-point tele-conference systems, the recognition of an asker's identity is an important task. mainly involving two factors. One is to provide the identity information of the asker to the speaker in a remote place where he can not see the askers. The other is to serve the conference recording and indexing system. which is a subsystem of the smart multi-point tele-conference system. The object of the conference recording and indexing system is to provide an information acquisition tool to persons who are late for the conference or will not attend the conference. Who has given a talk? What's the talk about? Who has asked questions? What are the questions? and so on. All of these will be recorded into an information database according to the time, the speakers' and askers' names, the content of the talk and so on [1].

We plan to use voice and face recognition techniques to recognize the identities of the askers, but in this paper we only consider face recognition techniques. As a person identity identification technique, many lace recognition algorithms have been proposed [2|[3][4][5] depending on face feature extraction and classification methods. This paper will introduce a new face recognition algorithm based on wavelet transform and ANN classification algorithm.

The rest of the paper is composed of live sections. Section 2 introduces the smart multi-point tele-conference system being studied in MIC, $\Lambda$ TR. Section 3 mainly focuses on the detection of the "ask action". Section 4 deals with the identification of the askers by using wavelet transform. Section 5 discusses experimental results. Section 6 concludes the paper.

\section{Smart multi-point tele-conference}

The smart multi-point tele-conference system heing studied at MIC, $\triangle T R$ is a multi-point tele-conference system whose object is to construct an intelligent system with the following smart properties [1]: (1) It can recognize the identity of the speaker automatically: (2) It can record and index the content of the speaker's talk automatically: (3) It can recognize the identity of the asker and record the content of the asker's question automatically: (4) It can recognize the reaction of the audience automatically: (5) The system can count the number of the attendee automatically.

The smart multi-point tele-conference system is mainly composed of three subsystems corresponding to the smart properties. Besides the conference recording and indexing system mentioned above, there are two other subsystems: one is a recognition subsystem of the audience's reaction, and the other is a state analysis system of the speaker. The task of the recognition subsystem of the audience's reaction is to collect the visual information from the audience and use it to detect. recognize and evaluate the behaviors and activities of the audience, for example, whether the audience is paying attention to the speaker or not, whether the audience agrees with the speaker or not, whether the audience feels hored or not, and so on. Obviously, this system is very useful not only to the speaker, but also to the audience. The task of the state analysis system of the speaker is to apply audio and visual information to recognize who is the speaker and determine the state of the speaker, i.e., fatigue, excitement and so on. This subsystem provides audio and visual information and the processing results to the audience as well as to the speaker himself

\section{Detection of "Ask action"}

In multi-point tele-conferences, the detection of "ask" actions (here an "ask" action is assumed to be "putting up a hand") is very important because this helps the speaker understand whether listeners have questions, how many listeners have questions, or if she/he should stop to answer questions.

The "ask " action detection processor in our system is described in Figure 1. First, the image captured from some site is fed into a color-based hand detection processor that is used to detect the skin color regions from the complex background and to separate the hand regions from face skin color regions (we suppose that the skin color regions are only composed of hand and face skin color regions) [6]. The hand information is then sent to the hand direction estimator, which estimates the hand direction angle. The estimation involves the following steps. First, the pixel values of hand skin region are binarized into two values, 1 and $I$ (after being binarized, the 
image is denoted by image $(x, y))$. The binarization is implemented by setting the pixel values in the hand region to 1 ,
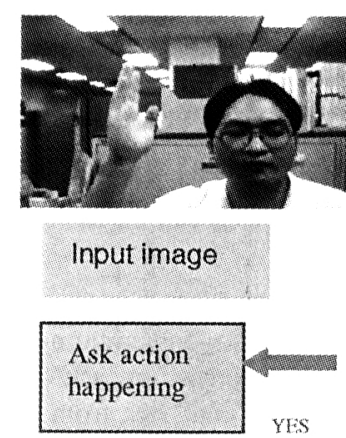

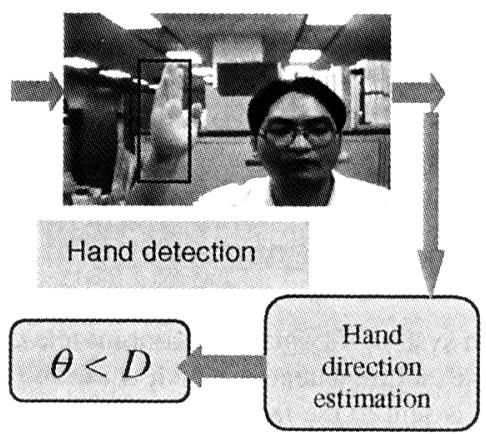

Figure 1. "Ask" action detection algorithm

and by setting the pixel values to 0 in the other regions Second, calculations are made with the following formulas

$$
\begin{aligned}
& F_{x x}^{2}=\sum_{y=0}^{N_{v}} \sum_{x=0}^{N_{v}}\left(x-c_{v}\right)^{2} * \text { image }(x, y) \\
& F_{v y}^{2}=\sum_{y=0}^{N_{v}} \sum_{x=0}^{N_{v}}\left(y-c_{y}\right)^{2} * \operatorname{image}(x, y) \\
& F_{v i}^{2}=\sum_{v=1}^{N} \sum_{v=1}^{N}\left(x-c_{v}\right) \times\left(y-c_{v}\right)^{2} * \operatorname{image}(x, y) \\
& \tan (2 \alpha)=\frac{2 F_{x y}^{2}}{F_{x, x}^{2}-F_{y y}^{2}}
\end{aligned}
$$

where $N_{x}, N_{y}$ are the width and height of the image and $\left(c_{x}, c_{y}\right)$ is the center of the hand, which is obtained with the following formulas:

$$
\begin{aligned}
& C_{x}=\frac{1}{N} \sum_{n=0}^{N} x \times \text { image }(x, y) \\
& C_{y}=\frac{1}{N} \sum_{n=0}^{N} y \times \text { image }(x, y)
\end{aligned}
$$

Here $\mathrm{N}$ is the number of the pixels with value $\mathrm{I}$ of the binarized image.
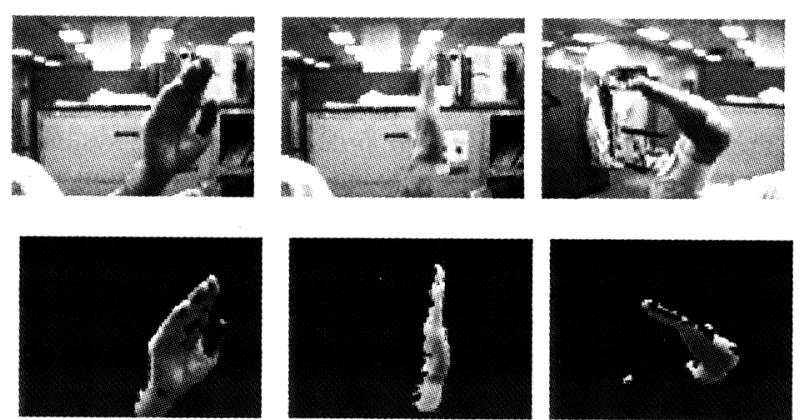

(a) $\theta=25.444132$ (c) $\theta=63.943481$
Figure 2. Estimation of hand direction (degree)
Third, the direction angle is estimated by

$$
\theta=\left|\alpha-\frac{\pi}{2}\right|
$$

A threshold D (25 in our system) is set in advance to decide whether an "ask" action has happened. If one has happened, then the face in the image is segmented [6] and fed to the wavelet-transform-hased asker identification processor. Figure 2 shows some results of our hand direction estimation algorithm.

\section{Wavelet-Transform-Based-Asker Identification}

\subsection{System architecture}

Figure 3 shows the basic system architecture. It is composed of four steps. In the first step. a face detector is applied to find and locate the asker lace in the image [6]. In the second step. the asker's lace image is decomposed into different subbands hy using the wavelet filters. In the third step, a neural-networkhased classifier is used to classify the face image hy using one subband and output a decision score corresponding to the subband. Finally the scores from different subbands are fused to give the recognition result.

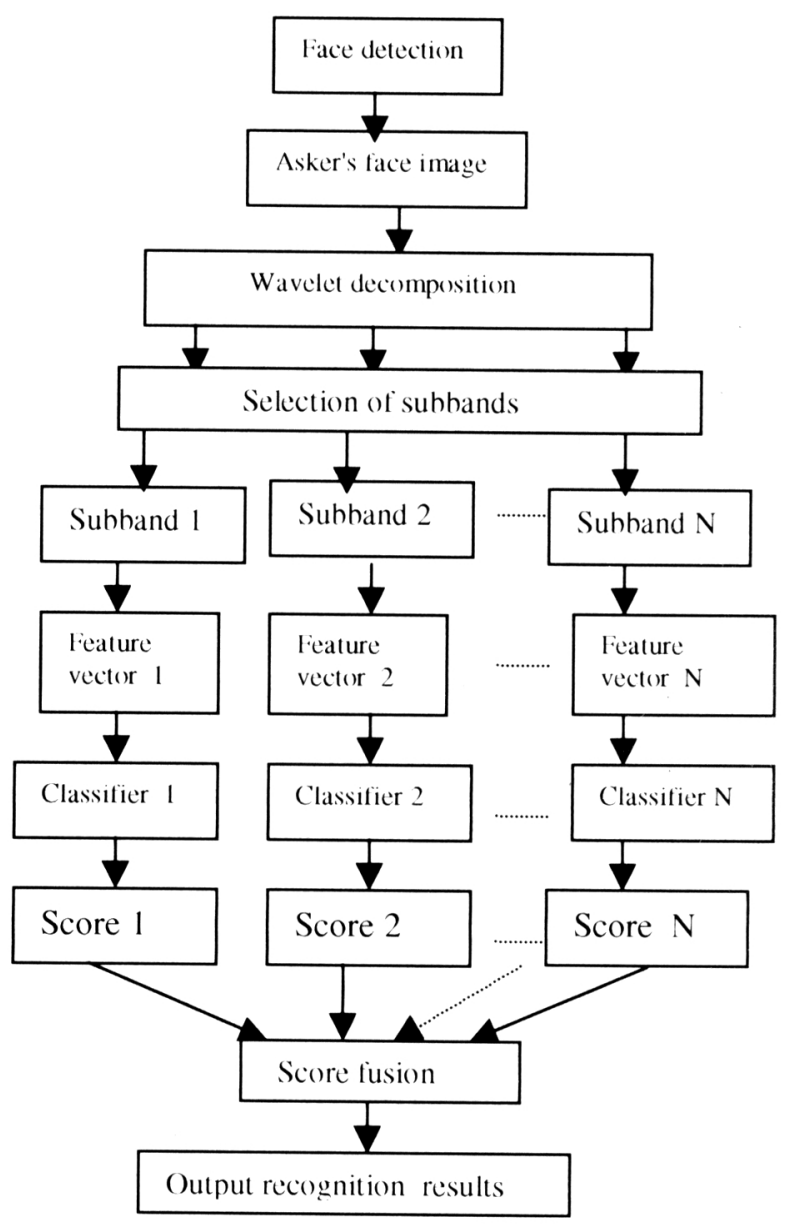

Figure 3. System architecture of asker recognition 


\subsection{Wavelet decomposition}

Here a simple description is given only, a systematic theory can be found in [7].

Let $P$ be the input tace image. Suppose that $H_{i}(Z) \quad(i=0,1)$ are low-pass filters, and $G_{i}(Z)$ $(i=0,1)$ are high-pass filters. The relationship among them can be described as follows:

$$
\begin{aligned}
& G_{0}(w)=e^{-j w} \overline{H_{1}(w+\pi)} \\
& G_{1}(w)=e^{-j w} \overline{H_{0}(w+\pi)} \\
& H_{0}(w) H_{1}(w)+\overline{H_{0}(w+\pi) H_{1}(w+\pi)}=1
\end{aligned}
$$

The wavelet decomposition of face image $P$ is shown in Figure 4 . Here subband $P_{1}$ corresponds to the smoothed. lowfrequency variations of the original image, subband $I_{1}$ corresponds to sharp changes in the vertical direction, subband $I_{2}$ corresponds to sharp changes in the horizontal direction and subhand $I_{3}$ corresponds to sharp changes in the nonhorizontal. nonvertical directions. The above procedure can be described hy the operator as follows:
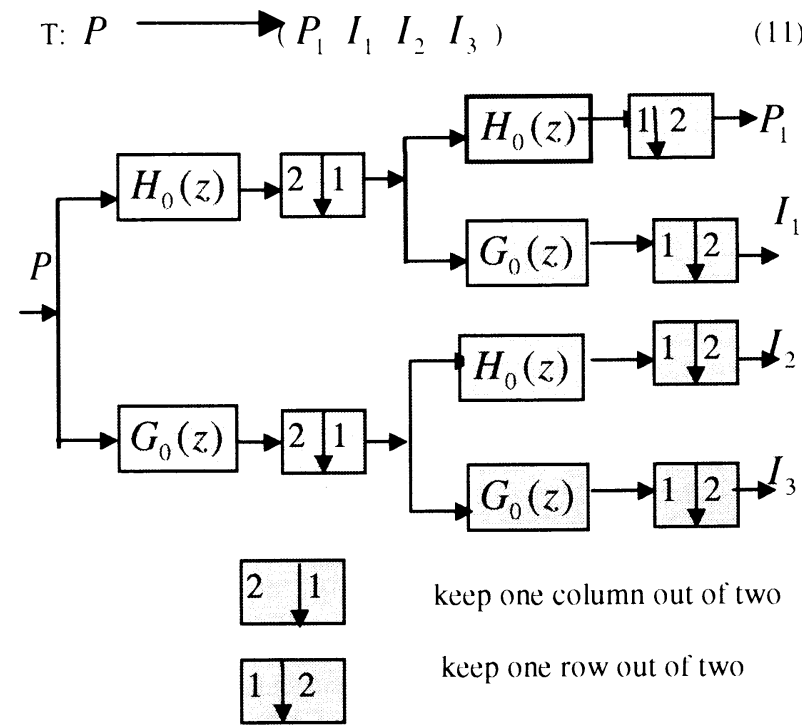

Figure 4. 2-1) Wavelet decomposition for image

By applying operator $\mathrm{T}$ to low frequency subband $P_{1}$, we obtain four other subhand components that possess similar properties to subbands $P_{1}, I_{1}, I_{2}, I_{3}$ respectively, denoted by $P_{2}, I_{1}\left(P_{1}\right), I_{2}\left(P_{1}\right), I_{3}\left(P_{1}\right)$. A similar processing can be continued until our need is met. After $\mathrm{N}$-times similar decomposition processing, we obtain the subband components shown below.
$\mathrm{T}: P \longrightarrow P_{1} I_{1} I_{2} I_{3}$
$\mathrm{T}: P_{1} \longrightarrow P_{2}, I_{1}\left(P_{1}\right), I_{2}\left(P_{1}\right), I_{3}\left(P_{1}\right)$

$\mathrm{T}: P_{N-1} \longrightarrow P_{N}, I_{1}\left(P_{N-1}\right), I_{2}\left(P_{N-1}\right), I_{3}\left(P_{N-1}\right)$

After the face image is decomposed into different subbands, the subbands used to classify the asker are selected from the low-frequency subbands $P_{1} \ldots \ldots, P_{N-1} P_{N}$. Figure 5 shows some subimages corresponding to these subbands.

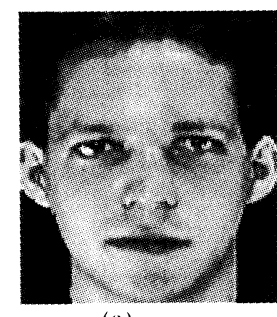

(a)

(a) Original image

(c) Subband $P_{2}$

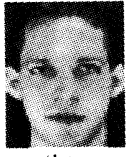

(b)

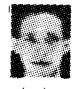

(c)

(b) Subband $P_{1}$

(d) Subhand $P_{3}$ m

(d)

Figure 5. An example of 3-level wavelet decomposition

\subsection{Neural Network Classifer}

Neural networks have been successfully applied to many classification problems. However, the conventional Multilayer perceptron uses an all-class-in-one-network structure and the number of hidden units is large, so many researchers use a oneclass-in-one-network structure [3] as shown in Fig.6. In this kind of neural networks, each subnet is trained individually and there is one subnet for one special person. The output of every subnet is () or 1. Before training, each person is allocated one subnet. If the input feature vector is from the person which the subset is allocated to, then the output of this subnet is set to be 1 , otherwise it is set to be (). The advantage of one-class-in-onenetwork in face recognition is that it is suitable for distributed computing. Therefore greater speedup can be anticipated [3].

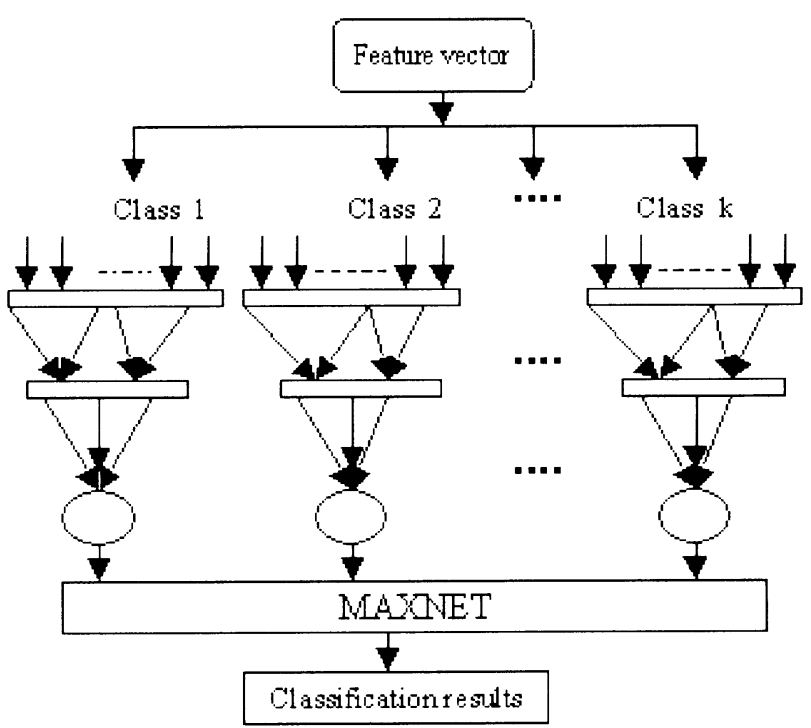

Figure 6. Structure of neural network face classification 


\subsection{Fusion algorithm}

The fusion algorithm in [8] is adopted. It is composed of two steps. The first step is to normalize the scores by using the following formula:

$$
S_{i, j}^{\prime}=\frac{1}{2}\left[\tanh \left(0.01 \frac{S_{i, j-u_{\text {tanh }}}}{\sigma_{\text {tanh }}}\right)+1\right] \in(0,1)
$$

where $\left\{S_{i, j}\right\}_{i=1, \ldots, I}$ is from classifier $\mathrm{j}$, and $\mathrm{I}$ is the number of people in the reference database. $u_{\text {tanh }}$ and $\sigma_{\text {tanh }}$ are the average and standard deviation estimates of the scores $\left\{S_{i, j}\right\}_{i=1, \ldots, I}$ as given by the Hampel estimators.

The normalized scores are fused with the following weighted geometric average:

$$
S_{i}=\left(\prod_{j} S_{i j}^{\prime w_{j}}\right)^{\frac{1}{\sum_{j} w_{j}}}
$$

where weight $w_{j}$ represents an estimate of the score dispersion in the right tail of the corresponding distributions:

$$
w_{j}=\frac{S_{i_{1}, j}^{\prime}-0.5}{S_{i_{2}, j}-0.5}-1.0
$$

where subscript index $i_{m}$ indicates the $m^{\text {th }}$ entry within the set of scores sorted by decreasing value.

\subsection{Choice of wavelet}

Biothogonal wavelets are chosen in our research. The biorthogonal wavelet filter is nine-seven taps, the impulse response coefficients for the analysis filters are shown in Table 1.

Table 1. Analysis biorthogonal wavelet filters

\begin{tabular}{|c|c|c|c|c|c|}
\hline Filter & $\sqrt{2} h_{0}$ & $\sqrt{2} h_{ \pm 1}$ & $\sqrt{2} h_{ \pm 2}$ & $\sqrt{2} h_{ \pm 3}$ & $\sqrt{2} h_{ \pm 4}$ \\
\hline & 0.852698 & 0.3774028 & -0.1106244 & -0.0238494 & 0.037828455 \\
\hline Filter & $\sqrt{2} g_{-1}$ & $\sqrt{2} g_{-2.0}$ & $\sqrt{2} g_{-3.1}$ & $\sqrt{2} g_{-4.2}$ & \\
\hline & 0.788485 & -0.4180923 & -0.0406894 & 0.064538882 & \\
\hline
\end{tabular}

\subsection{Classification Experiments}

The experiment used the Olivetti face database which contains 10) different images of each of 40) different persons. All the images were taken against a homogeneous background and some were taken at different times. The database includes frontal views of upright faces with slight changes in illumination, facial expression, with or without glasses. In our experiment, five training images and five test images of each person were used. The classification rate was $94.5 \%$. Compared with the algorithms [3] Eigenfaces (10\%) and Top-down $\operatorname{HMM}(13 \%)$, our algorithm performed better.
In order to test our algorithms, we have designed a simple networked smart multi-point conference system. The system is composed of five SGI workstations, with a digital camera mounted on each workstation.

The experiment was divided into two parts. In the first part, we tested the performance of our "ask" action detection algorithm. In the second part, we tested the performance of our asker identification algorithm.

We found that our "ask" action detection algorithm was very good while the asker identification algorithm was affected by light conditions, distances between the user and the computer, and poses of the detected face.

Therefore, in order to make our algorithm suitable for processing different conditions, after the asker's face is detected, we normalize the face. The normalization includes three processes: (1) normalization of the light condition. (2) normalization of the face pose, and (3) normalization of the face size. After normalization, the face is fed to the waveletbased face classification algorithm. The recognition rate was significantly improved.

We have tested our algorithm many times and experimental results show that our asker identification system can be applied to real multi-conference system to detect and recognize the askers.

\section{Conclusion}

In this paper, a wavelet-hased asker identification system was proposed for smart multi-point conference systems. In this system, hand direction is used to detect the "ask" action and wavelet is adopted to extract the feature for asker identification. Experimental results show that it is applicable.

\section{References}

[1] Jinshan Tang, " Research of Smart Teleconference system " , Research review, ATR, April, 2000.

[2] R. Brunelli and T. Poggio, "Face Recognition: Feature Versus Templates". IEEE Trans. On PAMI,15(10):1042-1052,1993.

[3] S.H.Lin,S.Y. Kung, and L.J.Lin."Face Recognition/Detection by Probabilistic Decision-Based Neural Network. "IEEE Trans. Neural Network, Vol. 8.Jan. 1997.

[4] C.W. Chen \& C.L Huang, "Human face recognition from a single front view". IEEE Transactions on Pattern Recognition and Machine Intelligence, 6, 571.1992.

[5] Y. Cheng, K. Liu, J. Yang, \& H Wang."A robust algebraic method for human face recognition.". Proceedings of the International Conference on Pattern Recognition. vol.2, The Hague, The Netherlands, p 221-224,1992.

[6] Jinshan Tang, Shinjiro Kawato,Jun Ohya, Ryohei Nakatsu "Locating Human Face in a Complex Background Including Nonface Skin Colors". The 2000) International Conference on Imaging Science, Systems, and Technology . June 26-29, 2000). Monte Carlo Resort, Las Vegas, Nevada. USA

[7] Mallat $S$. A theory for Multiresolution Signal Decomposition: The Wavelet Representation. IEEE Trans. On PAMI.,1 1(7):674-693. 1989. [8] D. Falavigna and R.Bruneli."Person recognition using acoustic and visual." Proceeding of ESCA workshop on automatic speaker recognition, identification and verification, Martigny, April 5-7, 71-74. 1994.

\section{Experiments in a real system}

